\title{
Predictors and Treatment Outcomes of Pediatric Osteosarcoma in Diverse Socioeconomic Backgrounds in Southeast Asia: A Retrospective Multicenter Study
}

\author{
Chalinee Monsereenusorn ${ }^{1 *}$, Ana Patricia Alcasabas ${ }^{2}$, Amos Hong Pheng \\ Loh $^{3,4}$, Shui Yen Soh ${ }^{4,5}$, Kenneth Wong Pak Leung ${ }^{6}$, Chetan Dhamne ${ }^{7}$, Sally \\ Blair $^{8}$, Catherine Lam ${ }^{8}$, Piya Rujkijyanont ${ }^{1}$, Chanchai Traivaree ${ }^{1}$, Apichat \\ Photia $^{1}$, Puwadon Veerapan ${ }^{9}$, Mark E Puhaindran ${ }^{10}$, Bernice L Z Oh ${ }^{11}$, Edward \\ H M Wang ${ }^{12}$, Carlos Rodriguez-Galindo ${ }^{8}$, Asian Childhood Cancer Alliance \\ Osteosarcoma Study Group
}

\begin{abstract}
Background: Pediatric osteosarcoma outcomes among developed and developing countries have not been previously compared. Countries in Southeast Asia (SEA) have a wide variety of socioeconomic statuses. A multi-institutional retrospective study was conducted to determine the prognostic factors and outcomes for pediatric osteosarcoma in SEA. Methods: Pediatric patients with osteosarcoma treated between 1998 and 2017 in 4 SEA pediatric oncology centers were studied. Countries were classified using the World Bank Atlas method. Kaplan-Meier method and Cox's Proportion Hazard Model were applied to estimate survival outcomes and identify prognostic factors. Results: In all, 149 patients with osteosarcoma with a mean age of $12.48 \pm 3.66$ years were enrolled. The localized to metastatic disease ratio was 1.5:1. The 5-year overall survival (OS) and event-free survival (EFS) were 53.8\% and 42\%, respectively. Prognostic factors associated with outcomes were country, stage of disease, MTX-containing regimens, and surgery type (p-value $<0.05)$. In patients with localized disease, EFS was superior with limb-salvage surgery $(62 \%)$ than amputation or rotationplasty (40\%) (p-value 0.009). MTX-containing chemotherapies provided higher OS (45.3\%) and EFS (37.9\%) than non-MTX regimens (12.3\% and 10.7\%, respectively) among metastatic patients (p-value 0.004 and 0.005 , respectively). Metastatic disease was an independent prognostic factor for death but not relapse outcome. Conclusion: The disease outcomes in SEA were acceptable compared to developed countries. The stage of disease was the only independent prognostic factor. MTX-containing regimens and limb-salvage surgery should be considered where possible.
\end{abstract}

Keywords: Osteosarcoma- Southeast Asia- metastasis- methotrexate- limb salvage

Asian Pac J Cancer Prev, 23 (2), 631-640

\section{Introduction}

Osteosarcoma is the most common primary malignant bone tumor among children and adolescents (Mirabello et al., 2009). Peak age incidence is the pubertal period, correlating to the pubertal growth spurt (Geller and Gorlick 2010; Luetke et al., 2014) with relatively high incidence in Asian/Pacific populations (Mirabello et al., 2009). Established prognostic factors include primary sites and size of tumors, surgery (Fu et al., 2020), metastatic

${ }^{I}$ Division of Hematology/Oncology, Department of Pediatrics, Phramongkutklao Hospital and Phramongkutklao College of Medicine, Bangkok, Thailand. ${ }^{2}$ Section of Hematology-Oncology, Department of Pediatrics, University of the Philippines - Philippine General Hospital, Manila, Philippines. ${ }^{3}$ Department of Pediatric Surgery, KK Women's and Children's Hospital, Singapore. ${ }^{4}$ Duke-NUS Medical School, Singapore. ${ }^{5}$ Haematology/Oncology Service, Department of Paediatric Subspecialties, KK Women's and Children's Hospital, Singapore. 'Department of Orthopaedic Surgery, KK Women's and Children's Hospital, Singapore. ${ }^{7}$ Department of Medical Oncology, Tata Memorial Hospital, Homi Bhabha National Institute, Mumbai, India. ${ }^{8}$ Department of Global Pediatric Medicine, St.Jude Children's Research Hospital, Memphis, TN, USA. ${ }^{9}$ Department of Orthopaedic Surgery, Phramongkutklao Hospital and Phramongkutklao College of Medicine, Bangkok, Thailand. ${ }^{10}$ Division of Muskuloskeletal Oncology, National University Hospital, Singapore. " Division of Pediatric Oncology, KTP University Childrens' Medical Institute, National University Hospital, Singapore. ${ }^{12}$ Department of Orthopaedics, Philippine General Hospital, Manila, Philippines. *For Correspondence: chalinee_monsereenusorn@pedpmk.org 
diseases, and tumor necrotic response after neoadjuvant chemotherapy (Abou Ali et al., 2019). Standard treatment for pediatric osteosarcoma in developed countries includes neoadjuvant methotrexate (MTX)-based chemotherapy followed by surgical control and subsequently adjuvant chemotherapy with desirable outcomes (Marina et al., 2016). However, in developing settings, non-MTX-based regimens predominate (Bajpai et al., 2017).

Management of osteosarcoma requires a complex multidisciplinary care team with advanced supportive care infrastructure (Othman et al., 2020). The outcomes of disease are distinct across territories. In countries with limited resources, outcomes are significantly poorer (Wiromrat et al., 2012) and most patients receive suboptimal treatment given chemotherapy shortage and unavailability of MTX plasma level monitoring (Choeyprasert et al., 2013; Choeyprasert et al., 2014), large tumor or late presentation (Puri et al., 2018), treatment abandonment, higher rates of metastasis (Friedrich et al., 2013), higher infection rates and disputative types of surgery (Qi et al., 2020).

Southeast Asia (SEA) consists of the ten countries which are defined as those with a gross national income (GNI) per capita in the year 2021, classified using the World Bank Atlas method in low (LIC), lower middle (LMIC), upper middle (UMIC) and high income (HIC) countries (The World Bank 2021). A marked diversity can be observed regarding socioeconomics and health care among SEA countries, from economic domination like Singapore to poorer nation status such as Cambodia (Chongsuvivatwong et al., 2011). The out-of-pocket expense discrepancy among countries is demonstrated. The out-of-pocket health expenditure range from 54\% in the Philippines (LMIC), 31\% in Singapore (HIC), to $11 \%$ in Thailand (UMIC) (The World Bank 2021). The economic status variables among these countries might affect a disparity in treatment and outcomes across the region.

The study of inter-ethnic variations in epidemiology, treatment, and outcomes of childhood osteosarcoma in SEA has been limited. Related reports have only addressed independent geographic areas (Noor et al., 2014; Pruksakorn et al., 2016). Accordingly, this study aimed to identify clinical characteristics, prognostic factors, and treatment outcomes as well as explore barriers to effective treatment of pediatric osteosarcoma in 4 oncology centers in SEA. The results of this study may lead to strategic treatment plans for the region which might improve the outlook for children with osteosarcoma among SEA.

\section{Materials and Methods}

\section{Patient selection}

The medical records of 208 pediatric patients with osteosarcoma who were newly diagnosed and treated primarily at four tertiary pediatric oncology institutions from three SEA countries between January 1, 1998, and December 31, 2017, were retrospectively reviewed. These centers were: Phramongkutklao Hospital, Bangkok, Thailand; Philippine General Hospital, Manila, the Philippines; KK Women's and Children's Hospital and
National University Hospital, Singapore.

The study's inclusion criteria included patients with osteosarcoma from birth to 21 years old whose diagnosis of osteosarcoma was confirmed by histology. Patients with uncertain diagnoses, incomplete medical records, lost to follow up and abandoned treatment were excluded from the study.

Written informed consent and assent waived. The study was approved by the Institutional Review Board, Royal Thai Army Medical Department according to the ethics principles of the Declaration of Helsinki (1975) and its revision (reference number: IRBRTA 1747/2561); SingHealth Centralized Institutional Review Board (2018/2750).

\section{Clinical definitions}

Treatment abandonment was defined as failure to initiate or to complete treatment. This excluded the decision of palliative treatment or discontinued treatment due to toxicity by primary oncologists.

Maximal tumor diameter (MTD) was defined as the largest diameter of the tumor, assessed by either computed tomography (CT), or magnetic resonance imaging (MRI) depending on institutional availability.

\section{Staging and disease evaluation}

Disease evaluation and treatment response were assessed using CT, MRI, or technetium (Tc)-99mmethylene-diphosphonate (MDP) bone scintigraphy depending on institutional availability. Patients were initially radiologically staged according to pulmonary and bone metastatic disease criteria from the European and American Osteosarcoma (EURAMOS)-I Study (Smeland et al., 2019) and imaging guidelines for children with Ewing sarcoma and osteosarcoma from the Children's Oncology Group (COG) Bone Tumor Committee, respectively (Meyer et al., 2008).

\section{Treatment}

Different treatment protocols were used according to institutional preference. The treatment process consisted of either surgery alone or combined with chemotherapy.

\section{Chemotherapy regimens}

Patients, regardless of localize or metastatic stage, were treated as per existing protocols based on institutional experts' experiences and chemotherapy availability. Chemotherapy protocols included non-MTX protocols including the European Organization for Research and Treatment of Cancer (EORTC) 80931 protocol (cisplatin and doxorubicin [CD]) (Lewis et al., 2007), St. Jude Children's Research Hospital Osteosarcoma-99 (OS-99) Trial (carboplatin, doxorubicin, and ifosfamide [CDI]) (Daw et al., 2011) and adapted OS-99 regimen adding etoposide (CDIE), and MTX-containing regimens including EURAMOS-1 regimen (MTX, doxorubicin and cisplatin [MAP] with additional ifosfamide and etoposide [MAPIE]) (Marina et al., 2016), and Italian Sarcoma Group study for Osteosarcoma (ISG/OS)-1 (MTX, doxorubicin, cisplatin, and ifosfamide [MAPI]) (Ferrari et al., 2014). 


\section{Surgery}

The surgical approach was indicated by the surgical experience in the individual institutions with curative or palliative intent. For limb-salvage surgery, resected bone was replaced with an implant, comprising either a bone graft or a metallic prosthesis.

\section{Outcome definition}

Overall survival (OS) was defined as the time duration from the date of diagnosis either to the time of death resulting from any causes or to the last follow-up for patients who survived.

Event-free survival (EFS) was defined as the time duration between the date of diagnosis and disease relapse, progression, or death, whatever came first, or the last follow-up for patients without events.

\section{Statistical analysis}

Overall demographic data, treatment strategies, and outcomes from all participating patients were analyzed using descriptive statistics, presented as mean with standard deviation (normal distribution) or median with range (nonnormal distribution) for continuous variables, and calculated using frequency and percentage for categorical variables. Countries were classified using the World Bank Atlas method (The World Bank 2021); the Philippines as LMIC, Thailand as UMIC, and Singapore as HIC and analyzed accordingly. Categorical and continuous variables were compared using Fisher's exact and One-Way ANOVA, respectively. Survival function was calculated using the Kaplan-Meier method and compared using Cox's Proportion Hazard Model. Cox's Proportion Hazard Model was used to evaluate the effect of covariates on hazard ratio (HR). Statistical and survival analyses were performed using STATA/IC, 16.0 Software, and a p-value $<0.05$ was considered statistically significant.

\section{Results}

\section{Patient characteristics}

Among the 208 patients with osteosarcoma eligible for the study, 59 (28\%) were excluded due to loss to follow-up or abandoned treatment. Therefore, 149 patients were subsequently enrolled in this study.

Among 149 patients with osteosarcoma enrolled in this study, $33(22.1 \%)$ patients were from Thailand (UMIC), $54(36.2 \%)$ patients from the Philippines (LMIC), and $62(41.6 \%)$ patients from Singapore (HIC). Patient characteristics including the age of diagnosis, MTD, primary sites, stage, sites of metastasis, chemotherapies, and surgery were analyzed according to the countries (Table 1).

The median age of diagnosis was 12.42 years. Median age of diagnosis among patients of the Philippines (14.42 years) was significantly older than Thailand (11.83 years) and Singapore (11.92 years) with p-value $<0.001$. Median MTD of patients from the Philippines $(15 \mathrm{~cm})$ was greater than patients of Thailand $(9.5 \mathrm{~cm})$ and Singapore $(7.75$ $\mathrm{cm}$ ) with p-value $<0.001$. Localized to metastatic disease ratio was 1.5:1. Nevertheless, the stage of disease did not significantly differ between the three countries. However, the metastatic sites were different between countries with p-value 0.013 .

In all, 144 (96.6\%) patients received chemotherapy and those receiving neoadjuvant chemotherapy totaled 131 (91\%) patients. However, neoadjuvant chemotherapy was more frequently delivered in Thailand $(n=33,100 \%)$ and Singapore $(n=57,95 \%)$ rather than the Philippines $(n=41,80.4 \%)$, with p-value 0.003 . Overall, half $(n=73$, $50.7 \%$ ) of patients received MTX-based chemotherapy, for which the majority was MAPIE $(n=40,27.8 \%)$. In addition, MTX-based regimens were applied in Thailand $(n=30,90.9 \%)$ and Singapore $(n=43,71.7 \%)$ while all patients in the Philippines received non-MTX protocols (p-value $<0.001$ ). CD regimen was the most commonlyused protocol among patients from the Philippines $(n=39$, $76.5 \%$ ).

Surgery was performed in $136(91.3 \%)$ patients. Nonetheless, limb salvage surgery was more frequently performed in patients from Thailand $(n=24,75 \%)$ and Singapore $(n=41,74.5 \%)$ than the Philippines $(n=9$, $18.4 \%$ ), with p-value $<0.001$.

\section{Overall treatment outcomes}

Five-year OS (Figure 1A) and EFS (Figure 1B) were
A

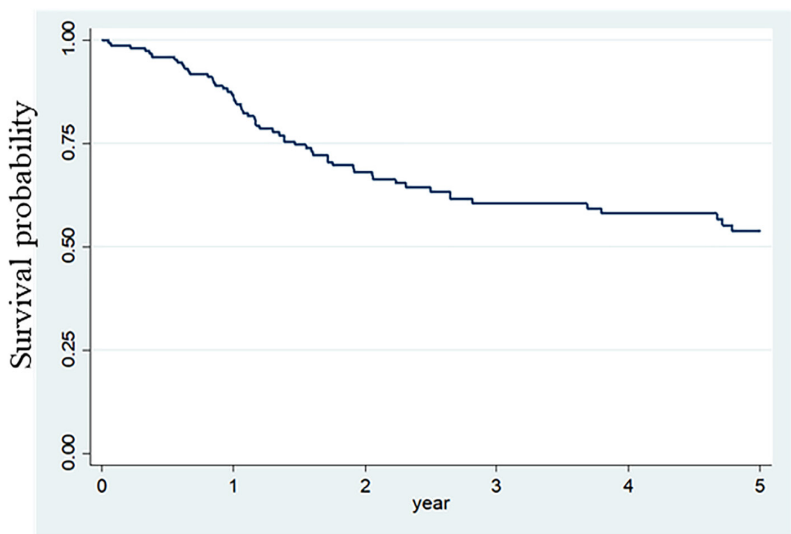

$\mathrm{B}$

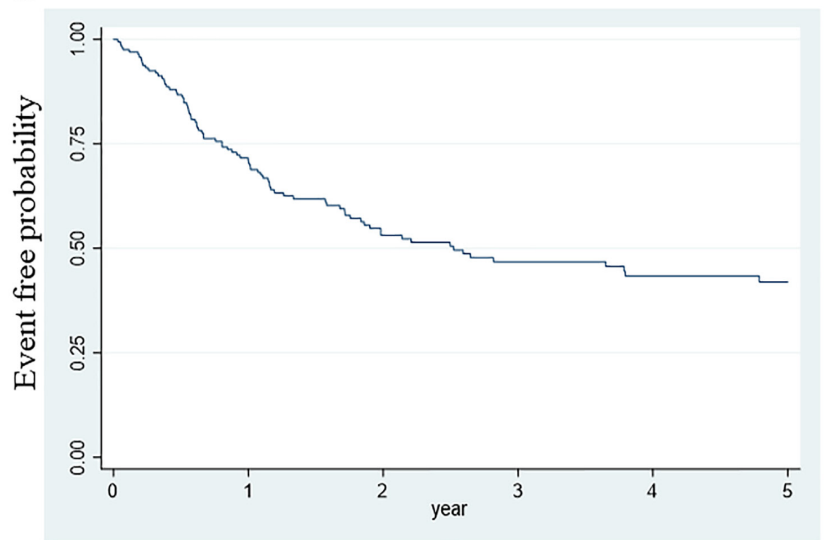

Figure 1. Overall Survival (A) and Event-Free Survival (B) of Pediatric Patients with Osteosarcoma (n=149). Note: Overall and event-free survival analysis was calculated using the Kaplan-Meier curves. 
Table 1. Patient Demographic Data $(n=149)$

\begin{tabular}{|c|c|c|c|c|c|}
\hline & $\begin{array}{c}\text { Thailand }(\mathrm{n}=33) \\
\mathrm{N}(\%)\end{array}$ & $\begin{array}{c}\text { The Philippines }(\mathrm{n}=54) \\
\text { N (\%) }\end{array}$ & $\begin{array}{c}\text { Singapore }(\mathrm{n}=62) \\
\mathrm{N}(\%)\end{array}$ & $\begin{array}{l}\text { Total }(\mathrm{n}=149) \\
\mathrm{N}(\%)\end{array}$ & $\mathrm{p}$-value \\
\hline Age of diagnosis (years) & & & & & $<0.001$ \\
\hline Mean \pm SD & $10.88 \pm 3.41$ & $13.91 \pm 3.15$ & $12.09 \pm 3.78$ & $12.48 \pm 3.66$ & \\
\hline Median (range) & $11.83(2.33-15.58)$ & $14.42(5.25-18.42)$ & $11.92(5.33-21.58)$ & $12.42(2.33-21.58)$ & \\
\hline \multicolumn{6}{|l|}{ Maximal tumor diameter $(\mathrm{cm})$} \\
\hline Mean \pm SD & $10.99 \pm 6.68$ & $17.64 \pm 11.34$ & $8.92 \pm 3.87$ & $13.46 \pm 9.45$ & $<0.001$ \\
\hline Median (range) & $9.5(2-38)$ & $15(5-82)$ & $7.75(2.2-16.5)$ & $12(2-82)$ & \\
\hline Primary site & & & & & 0.82 \\
\hline Femur & $16(48.4)$ & $30(55.5)$ & $29(46.8)$ & $75(50.3)$ & \\
\hline Tibia & $11(33.3)$ & $16(29.6)$ & $16(25.8)$ & $43(28.9)$ & \\
\hline Humerus & $2(6.1)$ & $6(11.1)$ & $10(16.2)$ & $18(12.1)$ & \\
\hline Fibula & $2(6.1)$ & $1(1.9)$ & $2(3.2)$ & $5(3.3)$ & \\
\hline Radius & $2(6.1)$ & $1(1.9)$ & $2(3.2)$ & $5(3.3)$ & \\
\hline Pelvis & $0(0)$ & $0(0)$ & $1(1.6)$ & $1(0.7)$ & \\
\hline Vertebrae & $0(0)$ & $0(0)$ & $1(1.6)$ & $1(0.7)$ & \\
\hline Skull & $0(0)$ & $0(0)$ & $1(1.6)$ & $1(0.7)$ & \\
\hline Stage & & & & & 0.053 \\
\hline Localized & $20(60.6)$ & $25(46.3)$ & $43(69.4)$ & $88(59)$ & \\
\hline Metastasis & $13(39.4)$ & $28(51.9)$ & $19(30.6)$ & $60(40.3)$ & \\
\hline Unknown & $0(0)$ & $1(1.8)$ & $0(0)$ & $1(0.7)$ & \\
\hline Metastatic site & & & & & 0.013 \\
\hline Lung & $10(76.9)$ & $26(92.9)$ & $12(63.2)$ & $48(80)$ & \\
\hline Bone & $3(23.1)$ & $0(0)$ & $2(10.5)$ & $5(8.3)$ & \\
\hline Combined & $0(0)$ & $2(7.1)$ & $5(26.3)$ & $7(11.7)$ & \\
\hline Chemotherapy & & & & & 0.072 \\
\hline Received & $33(100)$ & $51(94.4)$ & $60(96.8)$ & $144(96.6)$ & \\
\hline Not received & $0(0)$ & $3(5.6)$ & $0(0)$ & $3(2.1)$ & \\
\hline Unknown & $0(0)$ & $0(0)$ & $2(3.2)$ & $2(1.3)$ & \\
\hline Neoadjuvant chemotherapy & & & & & 0.003 \\
\hline Received & $33(100)$ & $41(80.4)$ & $57(95)$ & $131(91)$ & \\
\hline Not received & $0(0)$ & $10(19.6)$ & $3(5)$ & $13(9)$ & \\
\hline Chemotherapy regimen & & & & & $<0.001$ \\
\hline Non-methotrexate & $3(9.1)$ & $51(100)$ & $17(28.3)$ & $71(49.3)$ & \\
\hline $\mathrm{CD}$ & $3(9.1)$ & $39(76.5)$ & $12(20)$ & $54(37.5)$ & \\
\hline CDIE & $0(0)$ & $12(23.5)$ & $0(0)$ & $12(8.3)$ & \\
\hline CDI & $0(0)$ & $0(0)$ & $5(8.3)$ & $5(3.5)$ & \\
\hline Methotrexate-based & $30(90.9)$ & $0(0)$ & $43(71.7)$ & $73(50.7)$ & \\
\hline MAPIE & $9(27.3)$ & $0(0)$ & $31(51.7)$ & $40(27.8)$ & \\
\hline MAPI & $7(21.2)$ & $0(0)$ & $9(15)$ & $16(11.1)$ & \\
\hline MAP & $12(36.4)$ & $0(0)$ & $0(0)$ & $12(8.3)$ & \\
\hline Others & $2(6)$ & $0(0)$ & $3(5)$ & $5(3.5)$ & \\
\hline Surgery & & & & & 0.462 \\
\hline Surgery & $32(97)$ & 49 (90.7) & 55 (88.7) & $136(91.3)$ & \\
\hline No surgery & $1(3)$ & $5(9.3)$ & $3(4.8)$ & $9(6)$ & \\
\hline Unknown & $0(0)$ & $0(0)$ & $4(6.5)$ & $4(2.7)$ & \\
\hline Type of surgery & & & & & $<0.001$ \\
\hline Limb salvage & $24(75)$ & $9(18.4)$ & $41(74.5)$ & $74(54.4)$ & \\
\hline Amputation & $8(25)$ & $35(71.4)$ & $14(25.5)$ & 57 (41.9) & \\
\hline Rotationplasty & $0(0)$ & $5(10.2)$ & $0(0)$ & $5(3.7)$ & \\
\hline
\end{tabular}




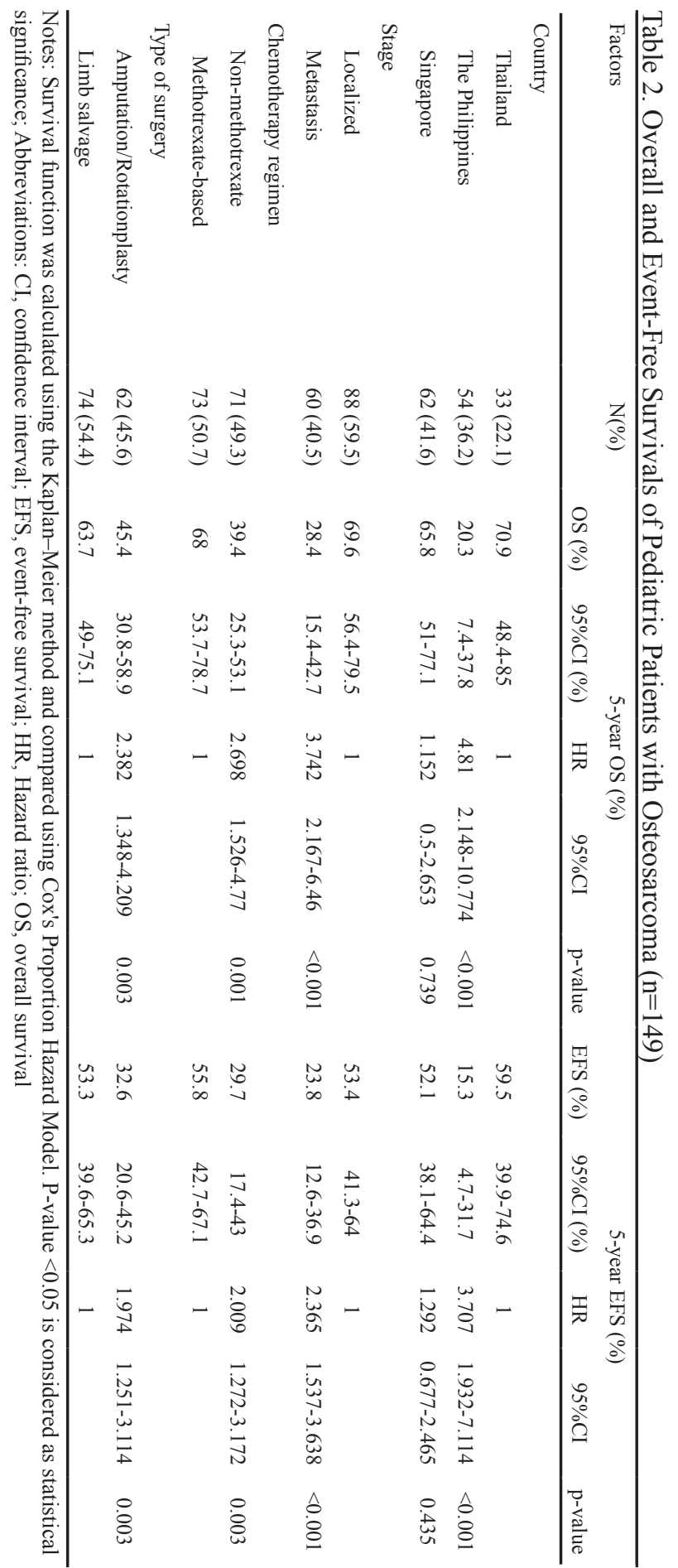

$53.8 \%$ (95\% confidence interval [CI], $43.9 \%$ to $62.7 \%)$ and $42 \%$ (95\% CI, $33.1 \%$ to $50.6 \%$ ), respectively. Relapse or disease progression occurred among 59 patients (39.6\%). Twenty-two (37.3\%) patients experienced disease progression while receiving treatment, 33 (55.9\%) patients had a recurrence of disease after the end of treatment, and 4 (6.8\%) patients showed unknown timing of disease recurrence. At the end of the study, 87 (58.4\%) patients were alive, with $74(85.1 \%)$ patients surviving without disease and $13(14.9 \%)$ patients living with disease. Sixty-two (41.6\%) patients expired. Fifty-one $(82.3 \%)$ patients died from disease progression or relapse and $11(17.7 \%)$ patients died from other causes including infection, electrolytes imbalance, secondary leukemia and cardiomyopathy.

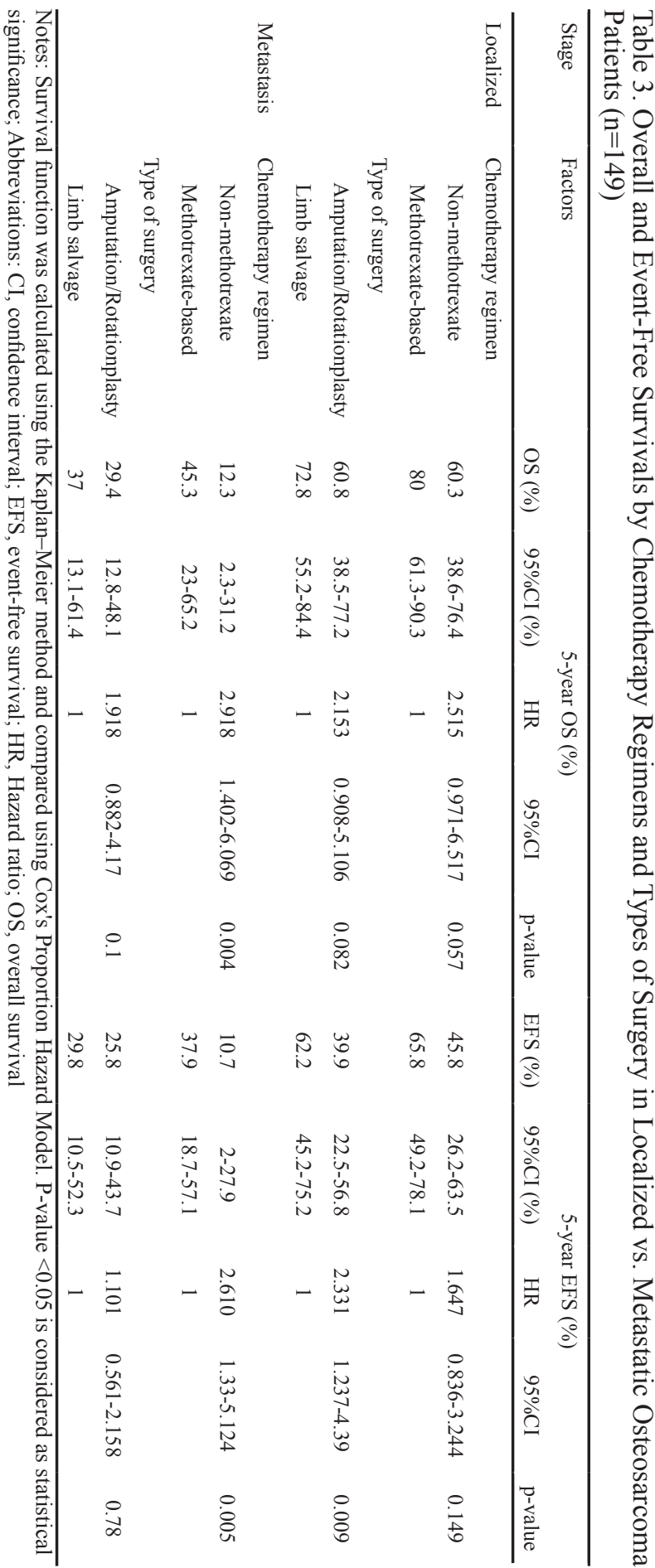

Five-year OS (Figure 2A) and EFS (Figure 2B) of patients from Thailand and Singapore did not significantly differ. Compared to Thailand (OS of 70.9\% and EFS of $59.5 \%$ ), patients from the Philippines had a significantly lower 5-year OS of 20.3\% (p-value $<0.001$ ) and EFS of $15.3 \%$ (p-value $<0.001$ ) as shown in Figure 2.

Five-year OS of localized and metastatic patients were $69.6 \%$ versus $28.4 \%$ with $p$-value $<0.001$, and EFS were $53.4 \%$ versus $23.8 \%$ with $p$-value $<0.001$.

Regarding chemotherapy regimens, 5-year OS of MTX and non-MTX-based regimens were $68 \%$ versus $39.4 \%$ with p-value 0.001 , and EFS were $55.8 \%$ versus $29.7 \%$ with p-value 0.003 .

Five-year OS of patients undergoing limb salvage surgery and amputation or rotationplasty were $63.7 \%$

Asian Pacific Journal of Cancer Prevention, Vol 23 


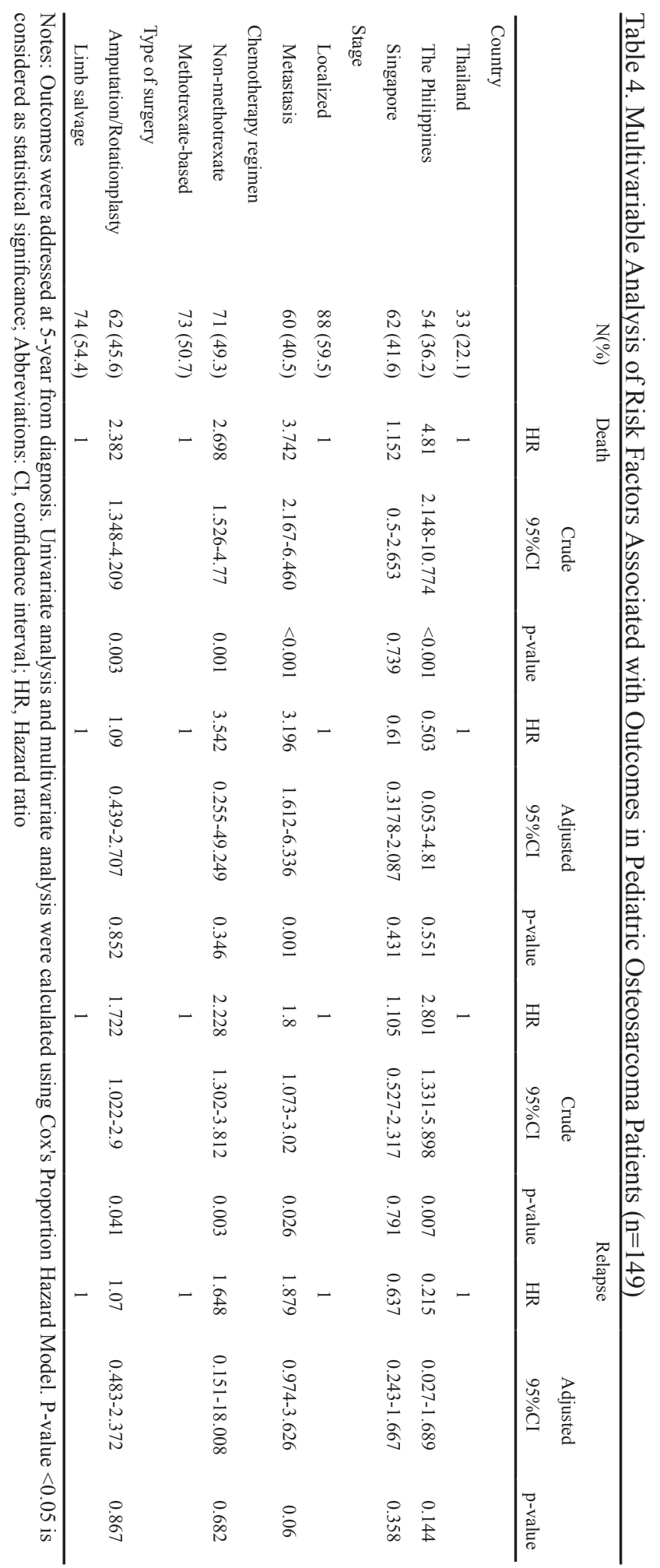

versus $45.4 \%$ with p-value 0.003 , and EFS were $53.3 \%$ versus $32.6 \%$ with p-value 0.003 . OS and EFS among patients with osteosarcoma according to country, stage of disease, chemotherapy regimens, and types of surgery are summarized in Table 2.

\section{Outcomes of localized vs. metastatic osteosarcoma} patients

Among patients with localized disease, 5-year OS did not differ according to MTX-based chemotherapy regimens or nature of surgery. However, the EFS of patients experiencing limb salvage surgery (62.2\%) was significantly higher than those with amputation or rotationplasty $(39.9 \%)$ with p-value 0.009 .

In patients with metastatic disease, those receiving MTX-based regimens had significantly higher 5-year OS and EFS (45.3\% and $37.9 \%$, respectively) than patients without MTX (12.3\% and $10.7 \%$, respectively) with p-value 0.004 and 0.005 , respectively.

However, OS and EFS did not differ significantly between metastatic patients experiencing limb salvage surgery and amputation or rotationplasty. Outcomes between localized versus metastatic osteosarcoma patients were described in Table 3.

\section{Factors associated with outcomes in pediatric} osteosarcoma

Countries, stage of disease, chemotherapy protocols, and types of surgery were significantly associated with death and relapse outcomes at univariate analysis with p-values $<0.05$. On subsequent multivariate analysis, disease stage remained the only independent risk factor significantly associated with survival outcome with p-value 0.001 (adjusted HR, 3.196; 95\% CI, 1.612 to 6.336) (Table 4).

\section{Discussion}

This study represents the first multicenter study of prognostic factors and associated outcomes of pediatric osteosarcoma in SEA, which is a unique geopolitical region with countries of diverse socioeconomic status, resulting in markedly differing management strategies and outcomes. Even though the data only included one country from each category and was limited to middle- and HIC, each country might represent the reality of countries in the same country classification. Few studies have evaluated treatment outcomes in low and middle income countries (LMC) such in the Asia-Pacific (Noor et al., 2014; Abou Ali et al., 2019; Bajpai et al., 2019) and in South Africa (Vasquez et al., 2016).

Osteosarcoma is the most common musculoskeletal malignancy with a high rate in Asian/Pacific Islander populations at the mean age of 13 years in this study which was similar to related studies in a developing country (Pruksakorn et al., 2016) or North America (Mirabello et al., 2009). Treatment abandonment is a major barrier in achieving desirable outcomes in LMC. Interestingly, MTD, especially among LMIC patients, was extremely large (Puri et al., 2018). This finding was likely related to delayed diagnoses, one of the crucial barriers to effective treatment of pediatric solid tumors (Loh et al., 2009). Diagnostic delay has also been associated with older age at diagnosis and nature of first local healthcare contact (Loh et al., 2012), which are significant factors that impact outcomes. Moreover, among the Philippines patients, we found a higher incidence of metastatic disease at diagnosis - a factor that may have further contributed to their observed outcomes.

Some limitations were encountered in terms of resource availability, supportive care contexts (Rastogi et al., 2018) as well as disease burdens including tremendous tumors or metastatic disease in a resource-constrained 
A

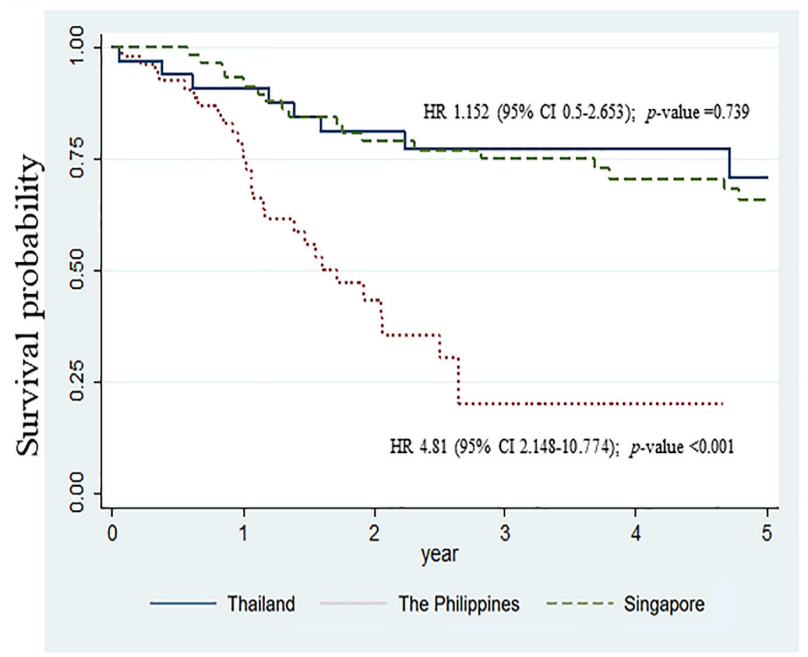

B

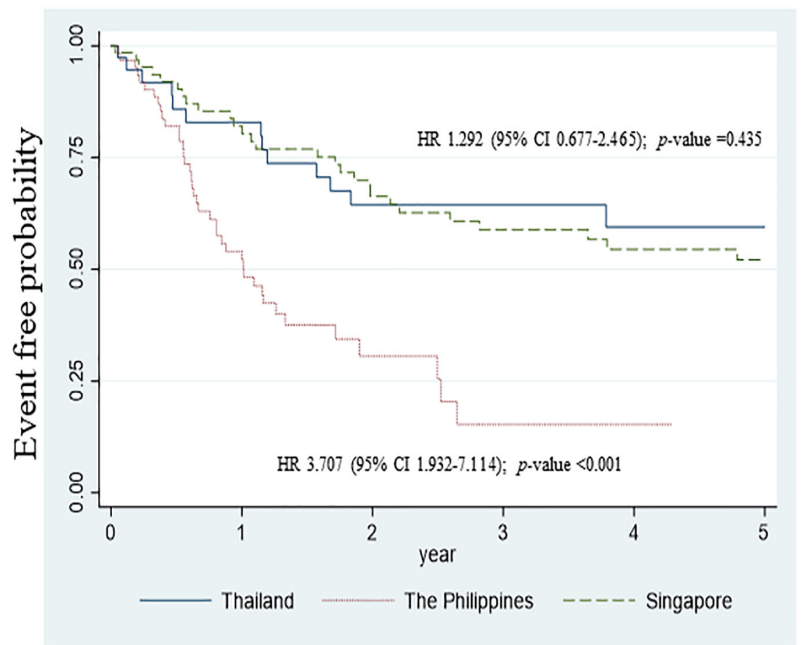

Figure 2. Overall Survival (A) and Event-Free Survival (B) of Pediatric Patients with Osteosarcoma ( $\mathrm{n}=149$ ) According to Countries. Notes: Survival function was calculated using the Kaplan-Meier method and compared using Cox's Proportion Hazard Model. P-value $<0.05$ is considered as statistical significance. Abbreviations: CI, confidence interval; HR, Hazard ratio

setting. These limitations challenge the local experts to adapt treatment from standard treatment to upfront surgery, followed by adjuvant chemotherapy.

MTX-based regimens have been shown data to substantially improve outcomes in pediatric osteosarcoma (Bacci et al., 1993). Although high dose MTX protocols have become the standard of treatment for pediatric osteosarcoma in developed countries, yet non-MTX containing regimens may still be more suitable and tolerable with desirable outcomes (Daw et al., 2011) especially in resource-constrained countries (Choeyprasert et al., 2013; Bajpai et al., 2017). MTX may associate with an increased admission rate and higher cost of treatment which lead to treatment abandonment (Verma et al., 2021). Therefore, non-MTX-containing regimens may be preferred in centers without MTX monitoring capabilities or bed occupancy limitations, but an assessment of risks and benefits is required to identify optimal treatment approaches to achieve the best outcomes in such settings.

Amputation is typically preferred over limb salvage for patients with older age, advanced stage, large tumor size, comorbidities and low socioeconomic status (Evans et al., 2020), such as in LMC. In addition, amputation is more often performed among metastatic patients in LMIC (Pakos et al., 2009; Noor et al., 2014). While significantly superior outcomes were observed in our patients who underwent limb salvage surgery than amputation, this finding may be confounded by the improved supportive care and adjuvant therapy in Singapore and Thailand in our study (Evans et al., 2020; Qi et al., 2020). Nonetheless, amputation may still be appropriate in resource-limited contexts, as it effectively lowers the rate of local recurrence (Nakamura et al., 2020) while preserving adequate functional outcomes and quality of life (Solooki et al., 2018).

Although the outcomes for pediatric osteosarcoma in SEA seemed to be comparable to that of developed countries, this would be optimistic due to the high rate of abandonment that was not incorporated in survival analysis. Notably, 5-year OS was closed to 5-year EFS, especially in metastatic patients in this study. The observation is likely related to being unable to achieve disease remission after encountering disease relapse and experiencing treatment-related toxicity including post-chemotherapy electrolyte disorders and infection. Increased treatment-related mortality (TRM) has been reported in patients treated in LMIC (Bajpai et al., 2019; Totadri et al., 2020) and receiving non-MTX regimens (Bajpai et al., 2019).

Outcomes between localized and metastatic osteosarcoma were diverse. The outcomes for localized osteosarcoma who completed treatment using CD and MTX containing regimens in SEA institutions were similar to long-term data from European Osteosarcoma Intergroup (Whelan et al., 2012) and EURAMOS (Smeland et al., 2019). MTX-based chemotherapy enhanced OS and EFS in metastatic diseases. Patients with metastatic disease experienced non-relapse mortality for which MTX-containing chemotherapy should be substituted to decrease the mortality rate. Toxicities from non-MTX chemotherapies included electrolyte imbalance (Daw et al., 2011), bone marrow suppression (Patel et al., 2002) and secondary leukemia. Ifosfamide and etoposide have been reported to increase the risk of non-hematological toxicity (Marina et al., 2016). However, limb salvage surgery was related to superior EFS among localized osteosarcoma patients. Although most patients suitable for limb salvage surgery probably had localized and smaller tumors amenable for this technique while patients with metastatic or large tumors needed to be amputated, alternative factors might be a reflection of surgical experience in limb salvage surgery or financial affordable of the family.

Univariate prognostic factors related to OS and EFS were countries, stage of disease (Fu et al., 2020), MTX-containing regimens and surgery types (Pakos 
et al., 2009), while the metastatic disease was the only independent prognostic factor for OS, but not relapse.

Therefore, the recommended treatment approaches for pediatric osteosarcoma in SEA should be tailored by disease stage. In localized disease, limb salvage surgery could be beneficial where possible. For metastatic disease, high dose MTX might be feasible (Choeyprasert et al., 2014) and tolerable to decrease treatment toxicity such as late effects of chemotherapy from alkylators and eventually decrease disease-associated mortality (Choeyprasert et al., 2014). Supportive care and appropriate hydration strategies can prevent side effects in a resource-constrained setting with limited capabilities to monitor plasma MTX level (Traivaree et al., 2018).

Strategies to improve treatment outcomes for pediatric patients with osteosarcoma in countries with limited resources include promoting early diagnosis, improving supportive care to reduce TRM (Yadav et al., 2014), increasing access and widening insurance coverage (Perez-Cuevas et al., 2013) for lowering abandonment, enhancing multidisciplinary care management (Friedrich et al., 2014), and encouraging more multi-institutional studies to address the treatment barriers (RodriguezGalindo et al., 2015).

\section{Limitations of the study}

This study was a retrospective study in which data collection might not have been uniform. The study included patients from three countries which might not represent the entire population of SEA, especially lack of LIC data. The diagnostic time which would associate with MTD, outcomes, and treatment abandonment was not explored. Data of grafts or prostheses among patients undergoing limb salvage surgery was largely unavailable. Different durations and heterogenicity of treatment could also have affected the outcomes.

In conclusion, the overall outcomes for pediatric patients with osteosarcoma in SEA were acceptable compared to developed countries, but abandonment should be taken into account. The stage of disease was the only independent prognostic factor to define survival but not recurrent outcomes. MTX-containing regimens are recommended to improve survival and prevent disease recurrence. Limb salvage is encouraged particularly among localized patients where possible. However, this advice on risk-adjusted determination on best surgical approach, depending on available surgical resources and financial capabilities.

\section{Author Contribution Statement}

CM conceptualized and designed the study, analyzed and interpreted data and was a major contributor in writing the manuscript. APA assisted with concept development and analyzed and interpreted data. AHPL, SYS assembled the data and assisted with data interpretation. SB served as an administrative supporter and coordinator. CL and CRG assisted in conceptualizing and designing the study. All authors contributed to patient care, collected data, and critically reviewed and approved the final manuscript.

\section{Acknowledgements}

The authors gratefully acknowledged the Asian Childhood Cancer Alliance (ACCA) on behalf of the St. Jude Global Alliance for supporting and coordinating this study.

Asian Childhood Cancer Alliance Osteosarcoma Study Group consists of Miriam Kimpo, Division of Pediatric Hematology/Oncology \& Bone Marrow and Cord Blood Transplantation, University Children's Medical Institute, National University Hospital, Singapore; Supak Cae-Ngow, Office of Research and Development, Phramongkutklao Hospital and Phramongkutklao College of Medicine, Bangkok, Thailand; Thidarat Meethawornkul, Department of Pediatrics, Phramongkutklao Hospital, Bangkok, Thailand; Sutipat Pairojboriboon, Department of Orthopaedic Surgery, Phramongkutklao Hospital and Phramongkutklao College of Medicine, Bangkok, Thailand; Kathleen J. Taleon, Eastern Visayas Regional Medical Center, Tacloban, Philippines; Julie Ritter, Department of Epidemiology and Cancer Control, St.Jude Children's Research Hospital, Memphis, TN, USA

\section{Ethics approval}

The study was approved by the Institutional Review Board, Royal Thai Army Medical Department according to the ethics principles of the Declaration of Helsinki (1975) and its revision (reference number: IRBRTA 1747/2561); SingHealth Centralized Institutional Review Board (2018/2750).

\section{Funding}

The funding to conduct the study was from the Phramongkutklao College of Medicine and Hospital, Royal Thai Army (CM); Children's Cancer Foundation (Singapore Childhood Cancer Registry) (SYS).

Scientific content approval

The manuscript was reviewed and approved by the Office of Research and Development, Phramongkutklao Hospital and Phramongkutklao College of Medicine, Bangkok, Thailand.

\section{Data sharing statement}

The datasets generated and/or analyzed during the current study are not publicly available due to privacy or ethical restriction. The data are available from the corresponding author upon reasonable request.

\section{Conflict of interest}

The authors declare that they have no competing interests.

\section{References}

Abou A, Salman BM, Ghanem KM, et al (2019). Clinical prognostic factors and outcome in pediatric osteosarcoma: Effect of Delay in Local Control and Degree of Necrosis in a Multidisciplinary Setting in Lebanon. J Glob Oncol, 5, 1-8.

Bacci GP, Picci S, Ferrari, et al (1993). Primary chemotherapy and delayed surgery for nonmetastatic osteosarcoma of the extremities. Results in 164 patients preoperatively treated with high doses of methotrexate followed by cisplatin and 
doxorubicin. Cancer, 72, 3227-38.

Bajpai JA, ChandrasekharanV, Simha, et al (2019). Osteosarcoma journey over two decades in India: Small steps, big changes. Pediatr Blood Cancer, 66, e27877.

Bajpai JA, ChandrasekharanV, Talreja, et al (2017). Outcomes in non-metastatic treatment naive extremity osteosarcoma patients treated with a novel non-high dosemethotrexatebased, dose-dense combination chemotherapy regimen 'OGS-12'. Eur J Cancer, 85, 49-58.

Choeyprasert W, Natesirinilkul R, Charoenkwan P, Sittipreechacharn S (2013). Carboplatin and doxorubicin in treatment of pediatric osteosarcoma: a 9-year single institute experience in the Northern Region of Thailand. Asian Pac $J$ Cancer Prev, 14, 1101-6.

Choeyprasert WS, Pakakasama N, Sirachainan, et al (2014). Comparative outcome of Thai pediatric osteosarcoma treated with two protocols: the role of high-dose methotrexate (HDMTX) in a single institute experience. Asian Pac J Cancer Prev, 15, 9823-9.

Chongsuvivatwong VKH, Phua M, Yap T, et al (2011). Health and health-care systems in southeast Asia: diversity and transitions. Lancet, 377, 429-37.

Daw NCMD, Neel BN, Rao et al (2011). Frontline treatment of localized osteosarcoma without methotrexate: results of the St. Jude Children's Research Hospital OS99 trial. Cancer, 117, 2770-8.

Evans DRA, Lazarides JD, Visgauss, et al (2020). Limb salvage versus amputation in patients with osteosarcoma of the extremities: an update in the modern era using the National Cancer Database. BMC Cancer, 20, 995.

Ferrari SC, Meazza E, Palmerini, et al (2014) Nonmetastatic osteosarcoma of the extremity. Neoadjuvant chemotherapy with methotrexate, cisplatin, doxorubicin and ifosfamide. An Italian Sarcoma Group study (ISG/OS-Oss). Tumori, 100, 612-9.

Friedrich PR, Ortiz S, Fuentes, et al (2014). Barriers to effective treatment of pediatric solid tumors in middle-income countries: can we make sense of the spectrum of nonbiologic factors that influence outcomes?. Cancer, 120, 112-5.

Friedrich PR, Ortiz K, Strait, et al (2013). Pediatric sarcoma in Central America: outcomes, challenges, and plans for improvement. Cancer, 119, 871-9.

Fu PY, Shi G, Chen, et al (2020). Prognostic factors in patients with osteosarcoma with the surveillance, epidemiology, and end results database. Technol Cancer Res Treat, 19, 1533033820947701.

Geller DS, Gorlick R (2010). Osteosarcoma: a review of diagnosis, management, and treatment strategies. Clin $\mathrm{Adv}$ Hematol Oncol, 8, 705-18.

Lewis IJMA, Nooij J, Whelan, et al (2007). Improvement in histologic response but not survival in osteosarcoma patients treated with intensified chemotherapy: a randomized phase III trial of the European Osteosarcoma Intergroup. J Natl Cancer Inst, 99, 112-8.

Loh A, Aung HL, Ha C, et al (2012). Diagnostic delay in pediatric solid tumors: a population based study on determinants and impact on outcomes. Pediatr Blood Cancer, 58, 561-5.

Loh A, HaJ HC, Chua H, et al (2009). Delays in diagnosis of pediatric solid tumors in Singapore. $J$ Pediatr Hematol Oncol, 31, 734-8.

Luetke A, Meyers PA, Lewis I, Juergens H (2014). Osteosarcoma treatment - where do we stand? A state of the art review. Cancer Treat Rev, 40, 523-32.

Marina NMS, Smeland SS, Bielack, et al (2016). Comparison of MAPIE versus MAP in patients with a poor response to preoperative chemotherapy for newly diagnosed high-grade osteosarcoma (EURAMOS-1): an open-label, international, randomised controlled trial. Lancet Oncol, 17, 1396-1408.

Meyer JSHR, Nadel N, Marina, et al (2008). Imaging guidelines for children with Ewing sarcoma and osteosarcoma: a report from the Children's Oncology Group Bone Tumor Committee. Pediatr Blood Cancer, 51, 163-70.

Mirabello L, Troisi RJ, Savage SA (2009). Osteosarcoma incidence and survival rates from 1973 to 2004: data from the Surveillance, Epidemiology, and End Results Program. Cancer, 115, 1531-43.

Nakamura TJ, Sugaya N, Naka, et al (2020). Standard treatment remains the recommended approach for patients with bone sarcoma who underwent unplanned surgery: Report from the Japanese Musculoskeletal Oncology Group. Cancer Manag Res, 12, 10017-10022.

Noor S, Thornormoethsson HS, Zervas CT, et al (2014). Limb versus life: the outcomes of osteosarcoma in Cambodia. Int Orthop, 38, 579-85.

Othman M, Blair YS, Nah SA, et al (2020). Pediatric Solid Tumor Care and Multidisciplinary Tumor Boards in Lowand Middle-Income Countries in Southeast Asia. JCO Glob Oncol, 6, 1328-45.

Pakos EEAD, Nearchou RJ, Grimer, et al (2009). Prognostic factors and outcomes for osteosarcoma: an international collaboration. Eur J Cancer, 45, 2367-75.

Patel SJJW, Lynch Jr, Johnson T, et al (2002). Dose-intense ifosfamide/doxorubicin/cisplatin based chemotherapy for osteosarcoma in adults. Am J Clin Oncol, 25, 489-95.

Perez-Cuevas RSV, Doubova M, Zapata-Tarres, et al (2013). Scaling up cancer care for children without medical insurance in developing countries: The case of Mexico. Pediatr Blood Cancer, 60, 196-203.

Pruksakorn DA, Phanphaisarn D, Pongnikorn, et al (2016) AgeStandardized Incidence Rates and Survival of Osteosarcoma in Northern Thailand. Asian Pac J Cancer Prev, 17, 3455-8.

Puri A, Byregowda SA, Gulia, et al (2018). A study of 853 high grade osteosarcomas from a single institution-Are outcomes in Indian patients different?. J Surg Oncol, 117, 299-306.

Qi LX, Ren Z, Liu, et al (2020). Predictors and survival of patients with osteosarcoma after limb salvage versus Amputation: A Population-Based Analysis with Propensity Score Matching. World J Surg, 44, 2201-10.

Rastogi S, Aggarwal A, Tiwari A, Sharma V (2018). Chemotherapy in nonmetastatic osteosarcoma: Recent Advances and Implications for Developing Countries. $J$ Glob Oncol, 4, 1-5.

Rodriguez-Galindo CP, Friedrich P, Alcasabas, et al (2015). Toward the cure of all children with cancer through Collaborative Efforts: Pediatric Oncology As a Global challenge. J Clin Oncol, 33, 3065-73.

Smeland SSS, Bielack J, Whelan, et al (2019). Survival and prognosis with osteosarcoma: outcomes in more than 2000 patients in the EURAMOS-1 (European and American Osteosarcoma Study) cohort. Eur J Cancer, 109, 36-50.

Solooki S, Mostafavizadeh Ardestani SM, Mahdaviazad H, Kardeh B (2018). Function and quality of life among primary osteosarcoma survivors in Iran: amputation versus limb salvage. Musculoskelet Surg, 102, 147-51.

The World Bank (2021). Out-of-pocket expenditure (\% of current health expenditure): World Health Organization Global Health Expenditure database. from https://data.worldbank. org/indicator/SH.XPD.OOPC.CH.ZS.

The World Bank (2021). World Bank Country and Lending Groups." from https://datahelpdesk.worldbank.org/ knowledgebase/articles/906519.

Totadri SD, Bansal KL, Rao N, et al (2020). Challenges in the management of localized Ewing sarcoma in a developing 
country. Pediatr Hematol Oncol, 37, 610-9.

Traivaree C, Likasitthananon N, Monsereenusorn C, Rujkijyanont P (2018). The effect of intravenous hydration strategy on plasma methotrexate clearance during intravenous high-dose methotrexate administration in pediatric oncology patients. Cancer Manag Res, 10, 4471-8.

Vasquez LF, Tarrillo M, Oscanoa, et al (2016). Analysis of prognostic factors in high-grade osteosarcoma of the extremities in children: A 15-Year Single-Institution Experience. Front Oncol, 6, 22.

Verma PS, Jain G, Kapoor, et al (2021). IAP chemotherapy regimen is a viable and cost-effective option in children and adolescents with osteosarcoma: A Comparative Analysis With MAP Regimen on Toxicity and Survival. J Pediatr Hematol Oncol, 43, 466-71.

Whelan JSRC, Jinks A, McTiernan, et al (2012). Survival from high-grade localised extremity osteosarcoma: combined results and prognostic factors from three European Osteosarcoma Intergroup randomised controlled trials. Ann Oncol, 23, 1607-16.

Wiromrat PA, Jetsrisuparb P, Komvilaisak, et al (2012). Incidence and survival rates among pediatric osteogenic sarcoma cases in Khon Kaen, Thailand, 1985-2010. Asian Pac J Cancer Prev, 13, 4281-4.

Yadav SPN, Rastogi G, Kharya, et al (2014). Barriers to cure for children with cancer in India and strategies to improve outcomes: a report by the Indian Pediatric Hematology Oncology Group. Pediatr Hematol Oncol, 31, 217-24.

\section{(ब) $(0 \otimes$}

This work is licensed under a Creative Commons AttributionNon Commercial 4.0 International License. 